\title{
Endogenous Axoplasmic Proteins and Proteins Containing Nuclear Localization Signal Sequences Use the Retrograde Axonal Transport/Nuclear Import Pathway in Aplysia Neurons
}

\author{
Robert Schmied, ${ }^{1}$ Cheng-Chun Huang, ${ }^{2}$ Xiao-Ping Zhang, ${ }^{1}$ Danicia A. Ambron, ${ }^{1}$ and Richard T. Ambron ${ }^{1}$ \\ 'Departments of Anatomy and Cell Biology and 'Ololaryngology, College of Physicians and Surgeons of Columbia \\ University, New York, New York 10032
}

\begin{abstract}
When the nuclear localization signal peptide (sp) of the SV 40 large $T$ antigen was coupled to human serum albumin (HSA), rhodaminated ( $r$ ), and microinjected into axons of Aplysia neurons in vitro, the rHSA-sp was conveyed through the axon to the cell body and then into the nucleus (Ambron et al., 1992). But since rHSA-sp is an artificial construct, we needed to determine whether naturally occurring nuclear proteins use this pathway. We therefore injected calf thymus histone $\mathrm{H}-1$ and Xenopus oocyte nucleoplasmin into axons. By $3 \mathrm{hr}$ both were retrogradely transported and targeted into the nucleus, though histone $\mathrm{H}-1$ less efficiently than rHSAsp or nucleoplasmin. In contrast, neither rHSA, nor rHSA conjugated to a peptide with a random distribution of basic amino acids, was transported or imported. To see if proteins that use the pathway remain intact, we coupled sp to HRP. When injected into varicosities, the HRP-sp was transported/ imported to the nucleus, where it was enzymatically active.

A key issue was to determine whether endogenous proteins use this pathway. Consequently, axoplasm was extruded from Aplysia nerves and the proteins were fractionated by size. SDS-PAGE and Western blots showed that two fractions contained proteins that were recognized by an affinity-purified antibody to sp: fraction 3 included sp83, and fraction 4 contained sp75. In addition, these two proteins were found in nuclei isolated from neurons. To assess transport, the total proteins in the fractions were rhodaminated and injected into varicosities. Fraction 3, but not fraction 4, contained protein that was transported through the axon to the nucleus. Collectively, these data indicate that endogenous proteins, perhaps sp83, use the retrograde transport/ nuclear import pathway. Such proteins could inform the nucleus of events in the periphery as in injury or learning and memory.
\end{abstract}

IKey words: retrograde axonal transport, nuclear import, axoplasm, signal sequence, Aplysia neurons, nucleoplas$\min ]$

\footnotetext{
Receivea Jan. 14, 1993; revised Apr. 2, 1993; accepted Apr. 13, 1993.

We are grateful to Marilyn Smedman for her excellent technical assistance and to Dr. John Pintar for comments on the manuscript. We also thank Drs. Karl Feldherr for providing samples of nucleoplasmin, Diane Garsetti for synthesizing the signal peptides, and Sindu Krishna for help with the confocal microscopy. R.S. was supported by Training Grant T32 AG 00189. This work was supported by NIH Grants NS-22150 and NS-26638.

Correspondence should be addressed to Richard T. Ambron, Department of Anatomy and Cell Biology, 1204 Black Building, College of Physicians and Surgeons, Columbia University, New York, NY 10032.

Copyright $(1993$ Society for Neuroscience $0270-6474 / 93 / 134064-08 \$ 05.00 / 0$
}

We recently describcd an intraccllular pathway in Aplysia ncurons that conveys proteins from the axon periphery through the axon to the cell body and then into the nucleus (Ambron et al., 1992). The pathway is formally composed of two processes, retrograde axonal transport and nuclear import. While studies of the latter have been taken to the molecular level (see reviews by Goldfarb, 1989; Garcia-Bustos et al., 1991; Nigg et al., 1991; Silver, 1991), our knowledge of retrograde axonal transport is rather limited (Vallee and Bloom, 1991) and derives largely from studies of material endocytosed at nerve terminals. The discovery of this novel pathway allows us to view retrograde transport from a very different perspective. We initially assembled a construct consisting of the nuclear localization signal peptide (sp) of the SV-40 large $\mathrm{T}$ antigen conjugated to rhodaminated human serum albumin (rHSA) (Lanford et al., 1986). When injected into varicosities on axons of Aplysia neurons regenerating in vitro, the rHSA-sp construct was rapidly transported through the axon exclusively in the retrograde direction. Transport could be hlocked by nocodazole, implying a dependence on microtubules (Ambron et al., 1992).

The fact that the construct gained access to retrograde transport from the axoplasm suggested that some soluble axonal molecules mimic the rHSA-sp and use the retrograde transport system to travel back to the cell body. It is significant that the sp provides access to retrograde transport as well as nuclear import. We base this idea on earlier experiments showing that whereas rHSA-sp was transported/imported, rHSA was neither transported nor imported, and rHSA coupled to a modified form of $\mathrm{sp}(\mathrm{msp}$ ) was transported and imported inefficiently (Ambron et al., 1992). We now provide direct evidence for the role of the peptide by showing that free sp prevents the transport of rHSAsp. Thus, we believe that $s p$ is the first signal to be identified that targets a protein for axonal transport. In addition, the ability of $s p$ to inhibit the movement of rHSA-sp implies that retrograde transport, like nuclear import (Crarcia-Bustos et al., 1991; Nigg et al., 1991; Silver, 1991), is a saturable process requiring specific receptors in the axoplasm.

While the previous studies broadly defined the pathway, several important issues needed to be addressed. First, rHSA-sp is an artificial construct in which as many as $20 \mathrm{sp}$ moieties are attached to a single molecule of HSA. Can naturally occurring nuclear proteins, which contain internal nuclear localization sequences (NLSs), also use this pathway? Here we show that they can. Xenopus oocyte nucleoplasmin, for example, is transported and imported almost as efficiently as rHSA-sp. Second, we did not determine whether proteins that use this pathway 
remain intact during transport and import; the movement of the rHSA-sp was judged solely by fluorescence. Finally, before we can begin to estimate the significance of the pathway to neurons, we have to identify the endogenous proteins that use the pathway. Several sp-containing proteins are present in the Aplysia nervous system (Ambron et al., 1992) and we now show that two of these, of 75 and $83 \mathrm{kDa}$, are present in axoplasm and the nucleus. In addition, the $83 \mathrm{kDa}$ polypeptide is in a fraction of axoplasm that exhibits transport/import after injection. Thus, the $83 \mathrm{kDa}$ constituent has the properties that one would expect for a protein that uses the pathway.

\section{Materials and Methods}

Animals. Adult Aplysia californica (100-250 gm), obtaincd from Marinus (Long Beach, CA), and juvenile animals, obtained from The University of Miami School of Marine and Atmospheric Sciences (Miami, FL), were maintained in circulating, aerated artificial seawater (Aquarium Systems, Eastlake, $\mathrm{OH}$ ) with seaweed as food.

Preparation of the nuclear import signal sequence constructs for injection. The sp (Lanford and Butel, 1984) was synthesized and purified as described (Ambron et al., 1992). Sp and the lysine/arginine peptide (LAp; Calbiochem, La Jolla, CA) were coupled to HSA (Calbiochem) or horseradish peroxidase (type VIA, Sigma, St. Louis, MO) with $n$-maleimido-benzoyl-hydroxy-succinimide (Pierce Chemicals, Rockford, IL) (Goldfarb et al., 1986), and the number of peptides/molecule protein was calculated from the change in migration after SDS-PAGE (Dworetzky et al., 1988).

Calf thymus histone $\mathrm{H}-1$ (Boehringer-Mannheim, Indianapolis, IN), nucleoplasmin (a gift from Dr. C. M. Feldherr, Univ. Florida), HSALAp, and HSA-sp were reacted with tetramethylrhodamine isothiocyanate (TRITC) (isomer R, Sigma) (Newmeyer et al., 1986) under identical conditions. The rhodamine:protein ratio in the constructs varied from 3.5 to 7.0. Unreacted TRITC was removed by gel filtration or dialysis.

Extrusion of axoplasm and isolation of proteins. The CNS of adult animals was excised intact and the right and left connectives and the pair of posterior pedal nerves were isolated. Axoplasm was extruded into iso-osmolar artificial seawater at $4^{\circ} \mathrm{C}$ (Sherbany et al., 1979). The extruded material was centrifuged at $125,000 \times g$ and the supernatant was processed on Centricon filters (Amicon, Beverly, MA) to obtain four fractions containing proteins of nominal $<10,10-30,30-100$, and $>100 \mathrm{kDa}$. The total proteins in each fraction were conjugated to TRITC and concentrated to about $10 \mu \mathrm{g} / \mu \mathrm{l}$ in injection buffer.

Isolation of nuclei. The cell bodies of large neurons, removed from the abdominal ganglion of adult animals, were placed in artificial seawater at $4^{\circ} \mathrm{C}$, and were manually dissected (Ambron, 1982). The bulk of the cytoplasm, which is bright orange, and the external envelope were removed and the nuclei were washed with medium until they were free of adherent cytoplasm. Intact nuclei were collected and broken by freezing and thawing, and a soluble fraction was obtained by centrifugation at $125,000 \times g$ (Elliot et al., 1993).

Growth and injection of neurons. Neurons, removed from the abdominal ganglion of juvenile Aplysia with a long segment of the original axon intact, were added to polystyrene dishes coated with polylysine and containing hemolymph:L15 (1:1) at $15^{\circ} \mathrm{C}$ (Schacher and Proshanski, 1983). Under these conditions the cut end of the axon seals over to form a large stationary varicosity from which neurites emerge (Ambron et al., 1992). Cells were injected during the second day of growth while their neurites were still elongating. Approximately $1 \mathrm{ng}$ of protein in $500-750 \mathrm{pl}$ potassium phosphate buffer ( $\mathrm{pH} 7.3$ ) was injected by pressure into the cell body or varicosity using micropipettes with $0.5 \mu \mathrm{m}$ tips fashioned with a Flaming, Brown electrode puller (Sutter Instrument Co., Novato, CA). After injection, cells were maintained at $15^{\circ} \mathrm{C}$, and then fixed with $4 \%$ paraformaldehyde in artificial seawater.

Confocal microscopy. Fixed juvenile cells were examined using a BioRad MRC-600 scanning laser confocal imaging system attached to a Leitz Ortholux microscopc. Cells were examined with a $25 \times, 0.75 \mathrm{NA}$ objective and the black-level settings, laser intensity, and aperture openings were the same in all the experiments. Within each experiment, background fluorescence in the nucleus of cells that were not injected was nulled by adjusting the gain. Background in the nucleus varied less than $10 \%$ from one experiment to the next. Iniected cells were then examined by fluorescence and bright-field microscopy. Optical sections through the varicosity, axon, cytoplasm, and nucleus were examined to assess fluorescence. Data were collected only from cells with approximately the same amount of fluorescence, and the source of the fluorescence was localized by reducing the gain. The noise level was minimized by averaging, using a Kalman filter, and the image was stored on a Bernoulli disk. An estimate of the amount of fluorescence in each region was obtained using the AREA program of the MRC 600 image processing system. Briefly, the fluorescent region of the axon was outlined and scanned, yielding an average fluorescence per unit area. This value was then multiplied by the volume occupied by the fluorescence. The process was repeated with the nucleus and the two values were used to compute the percent transport. Images were combined and transferred to a Sony video printer.

Horseradish peroxidase cytochemistry. Neurons in vitro were injected with HRP-sp. Three hours later they were fixed, washed, permeabilized with $0.1 \%$ Triton $\mathrm{X}-100$, and incubated in $0.2 \% 3,3^{\prime}$-diaminobenzidine (Sigma) in $50 \mathrm{~mm}$ Tris- $\mathrm{HCl}\left(\mathrm{pH} \mathrm{7.6)}\right.$ with the addition of $0.01 \% \mathrm{H}_{2} \mathrm{O}_{2}$ (Graham and Karnovsky, 1966). Noninjected neurons in the same dish had no staining of the nucleus.

$S D S-P A G E$ and Western blotting. Proteins were extracted into sample buffer containing SDS by heating at $70^{\circ} \mathrm{C}$ for $20 \mathrm{~min}$. Insoluble material was removed by centrifugation and the supernatant was electrophoresed on a $10 \%$ polyacrylamide gel according to Laemmli (1970). Polypeptides in the gel were either visualized by silver staining or transferred to nitrocellulose paper to be probed by an affinity-purified antibody to sp (Ambron et al., 1992).

\section{Results}

The sp provides access to the retrograde axonal transport portion of the transport/import pathway

Previous studies suggested that the sp is recognized by some component of the retrograde transport system (Ambron et al., 1992). To investigate the relationship between the sp and transport in greater detail, we microinjected terminal varicosities with proteins that contain an NLS and compared the extent to which they were transported. All these experiments were carried out under similar conditions: (1) four to nine cells were examined in each case, (2) the injection site was between 300 and $500 \mu \mathrm{m}$ from the cell body, (3) cells were injected with approximately the same amount of rhodaminated protein and data were collected only from cells with comparable levels of fluorescence, and (4) the distribution of fluorescence in the cell was determined by confocal microscopy so as to determine the source of the fluorescence and to distinguish unequivocally the nucleus from cytoplasm.

We first determined the time required for rHSA-sp to be transported to the nucleus. Six neurons were injected and in each the leading edge of the rHSA-sp traversed the axoplasm to the nucleus within about $20 \mathrm{~min}$. By $3 \mathrm{hr}$, even in neurons with the longest axons, the transport and import processes were complete (Fig. 1A). The two processes appeared tightly coupled since little rHSA-sp accumulated in the cytoplasm surrounding the nucleus. As previously reported (Ambron et al., 1992), the construct moved through the axon only in the retrograde direction and little remained at the injection site. During the course of these studies we injected both large and small varicosities and found that size had no influence on the rate or extent of transport. All subsequent studies were carried out on cells fixed $3 \mathrm{hr}$ after injection.

The HSA-sp used in these experiments contained from 2 to 20 peptides per molcculc of HSA with a mcan of about 12 . Evidently only a few peptides are needed to ensure retrograde transport since essentially all of the construct was transported to the cell body. This efficiency indicated that the peptide is recognized by a receptor and that the process should be satu- 

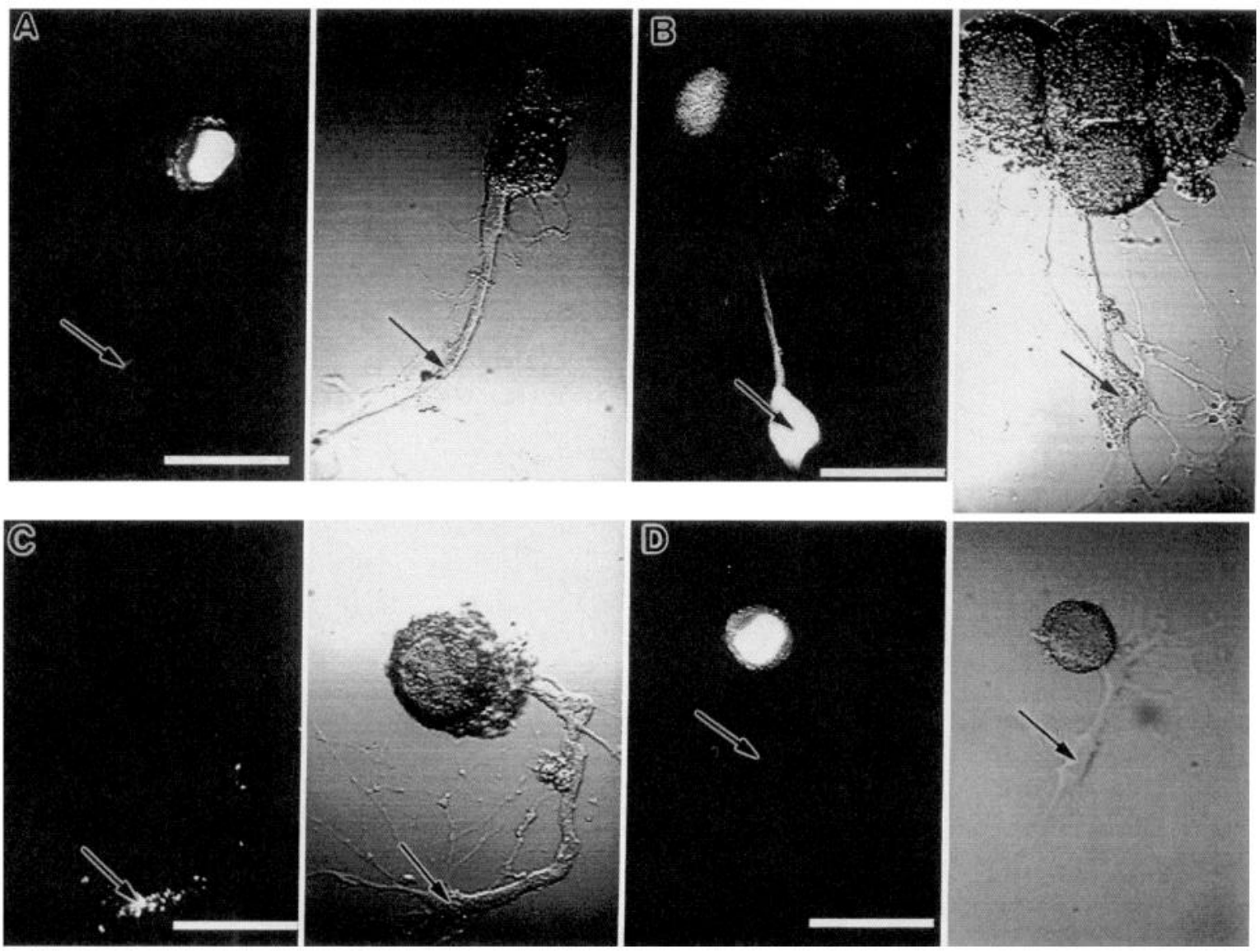

Figure 1. Distribution of fluorescent proteins $3 \mathrm{hr}$ after microinjection into varicosities. Cells were fixed and examined simultaneously by confocal fluorescence microscopy (left panels) and by bright-field microscopy (right panels). The nucleus of Aplysia neurons is large and often occupies much of the cell body. The arrows mark the injection site on the axon. A, rHSA-sp. All of the construct was transported retrogradely along the axon to the cell body and subsequently imported into the nucleus. None of the construct was transported in the anterograde direction into the growing neurites, many of which are out of focus. $B$, In contrast to the results in $A$, when the varicosity was injected with rHSA-sp plus a 50-fold excess of sp only a small amount of the rHSA-sp reached the nucleus; most was present in the varicosity and along the axon. The axon is fluorescent all the way to the cell body, but its proximal region is out of the plane of focus. Typical background fluorescence is seen in the nucleus and cytoplasm of the adjacent noninjected cells. C, rHSA-LAp. Coupling a basic peptide to the HSA did not result in any transport to the nucleus; all of the construct remained in the varicosity, where it was punctate. $D$, Rhodaminated nucleoplasmin injected into varicosities was transported efficiently along the axon and by $3 \mathrm{hr}$ was in the nucleus. Scale bars, $250 \mu \mathrm{m}$.

rable. To investigate this possibility we coinjected rHSA-sp and a 50 -fold molar excess of free sp into the varicosities of four neurons. Under these conditions, the extent of transport was markedly reduced and between $40 \%$ and $50 \%$ of the construct was now found at the terminal and along the axon (Fig. 1B). Experiments using a 25 -fold excess of the sp yielded similar results in three of five cells (data not shown). The finding that unconjugated sp competed with HSA-sp strengthens the idea that the sp moiety is required for transport.

The sp contains 10 amino acids with a cluster of basic residues (Table 1) that is characteristic of many proteins that are imported into the nucleus (Goldfarb et al., 1986; Lanford et al., 1986, 1990; Garcia-Bustos et al., 1991). To see if such a cluster is also important for retrograde transport, a 19 amino acid peptide containing randomly distributed lys/arg residues was conjugated to HSA (HSA-LAp; Table 1). After rhodamination, the rHSA-LAp was injected into the terminal varicosity. When observed $3 \mathrm{hr}$ later, all of the fluorescent construct was seen in a punctate pattern at the site of injection (Fig. 1C). A similar distribution was seen after injection of rHSA (Ambron et al., 1992). The rHSA-LAp had from two to six basic peptides per molecule of HSA and we would have seen movement of at least some of the construct had the LAp rendered HSA transportcompetent.

Since the sp units conjugated to HSA extend from the polypeptide backbone, the construct might not be a good model for endogenous proteins in which the signal sequence is an integral part of the polypeptide chain. We therefore injected the nuclear proteins histone $\mathrm{H}-1$ and nucleoplasmin into varicosities. $\mathrm{Nu}-$ cleoplasmin has two internal NLSs, one of which is similar to that of the sp, and histone $\mathrm{H}-1$ is highly basic with an sp-like sequence (Table 1). When the cells were examined $3 \mathrm{hr}$ after injection, both proteins had been transported, but not to the same extent. Four cells were injected with nucleoplasmin, and computations using an image analysis program showed that, on average, $93 \%$ of the fluorescent protein reached the cell body, where it was found in the nucleus (Fig. 1D). The rest was in the axon. Thus, nucleoplasmin was transported/imported almost as efficiently as rHSA-sp.

Histone $\mathrm{H}-1$, on the other hand, was transported relatively poorly (Fig. 2). We examined nine injected cells and in each found fluorescent protein along the axon (Fig. $2 A$ ). In seven of the cells some of the histone had reached the cell body and entered the nucleus where it was localized in patches (Fig. $2 B$ ). 


\begin{tabular}{|c|c|c|c|}
\hline Protein & Sequence $^{a}$ & $\begin{array}{l}\text { Transport } \\
\text { efficiency }^{b}\end{array}$ & $\begin{array}{l}\text { Nuclear } \\
\text { import }\end{array}$ \\
\hline HSA-sp & PKKKRK & ++++ & Yes \\
\hline HRP-sp & PKKKRK & ++++ & Yes \\
\hline HSA-msp & PKKTRK & + & Poor \\
\hline HSA-LAp & CSKARKQAASIKVAVSAAsxK & 0 & No \\
\hline HSA & - & 0 & No \\
\hline Histone $\mathrm{H}-1$ & PKKAKK & ++ & Yes \\
\hline Nucleoplasmin & RPAATKKAGQKKKKL & +++ & Yes \\
\hline Axoplasm & PKKKRK? & $?$ & Yes \\
\hline
\end{tabular}

a The sequence for histone $\mathrm{H}-1$ was obtained from Van Holde (1989), and that for nucleoplasmin, from Garcia-Bustos et al. (1991).

${ }^{b}$ The relative transport efficiency was based on the proportion of the protein that was conveyed back to the nucleus at $3 \mathrm{hr}$ after injection into the varicosity (see Results).

Image analysis indicated that only about $38 \%$ of the histone reached the nucleus. The histone in the axon at $3 \mathrm{hr}$ was not immobilized, however, and in two cells examined $24 \mathrm{hr}$ after injection almost all of the protein had entered the nucleus (not shown).

We followed fluorescence in these experiments and assumed
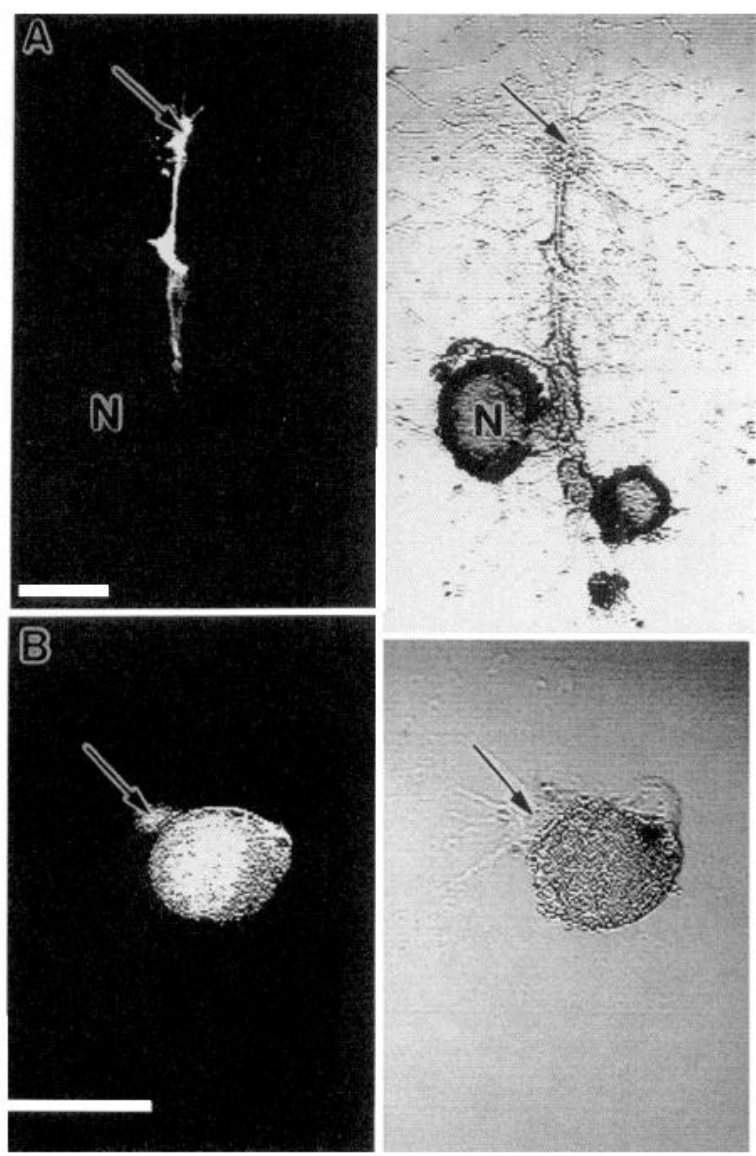

Figure 2. Distribution of rhodaminated histone $\mathrm{H}-13 \mathrm{hr}$ after injection. Nine neurons injected with histone $\mathrm{H}-1$ were examined by confocal fluorescence microscopy and bright-field microscopy. Unlike rHSA-sp and nucleoplasmin (Fig. 1), histone $\mathrm{H}-1$ was transported inefficiently. $A$, Most of the fluorescence was present along the axon and only a small amount reached the nucleus $(N)$, which is not in focus. $B$, In some injected cells a detectable amount of the histone reached the nucleus where it was distributed in patches. In this cell the axon grew under the cell soma and was not in the confocal plane. Scale bar, $250 \mu \mathrm{m}$. that the proteins remained intact during both transport and import. To see if this assumption was correct, we conjugated sp to the enzyme HRP. The HRP-sp was microinjected into the terminal varicosity of four cells and $3 \mathrm{hr}$ later the cells were assayed for activity. As can be seen in Figure 3, HRP reaction product was found in the nucleus. There was no staining in the nucleus of the noninjected cells (not shown). These results lead us to believe that enzymes and other proteins coupled to sp will retain their activity en route to the nucleus.

\section{Evidence that endogenous axoplasmic proteins use the transport/import pathway}

Polypeptides containing sp are present in axoplasm. An affinitypurified antibody to sp recognizes soluble polypeptides of 75 , 83,110 , and $>200 \mathrm{kDa}$ in the Aplysia nervous system (Ambron et al., 1992). Since we believe that sp-containing proteins use the transport/import pathway to communicate between the axon and nucleus, it follows that they would reside in axoplasm. Consequently, we extruded axoplasm from 20 Aplysia nerves, removing the organelles by centrifugation. The supernatant proteins were then separated by membrane exclusion filtration into four fractions nominally containing (1) $<10 \mathrm{kDa}$, (2) $10-30 \mathrm{kDa}$, (3) 30-100 kDa, and (4) $>100 \mathrm{kDa}$ proteins. A dot blot assay

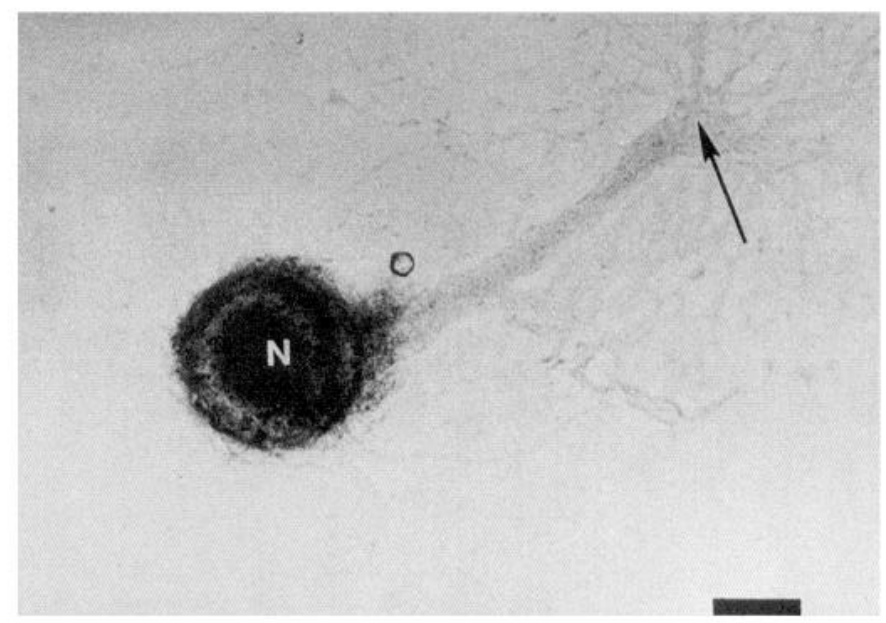

Figure 3. Coupling sp to HRP results in transport of the enzyme to the nucleus. The HRP-sp was injected into the varicosity (arrow) and $3 \mathrm{hr}$ later the cell was fixed and processed to detect HRP enzyme activity (see Materials and Methods). The results from a typical cell are shown. The nucleus $(N)$ is prominently stained. Scale bar, $100 \mu \mathrm{m}$. 


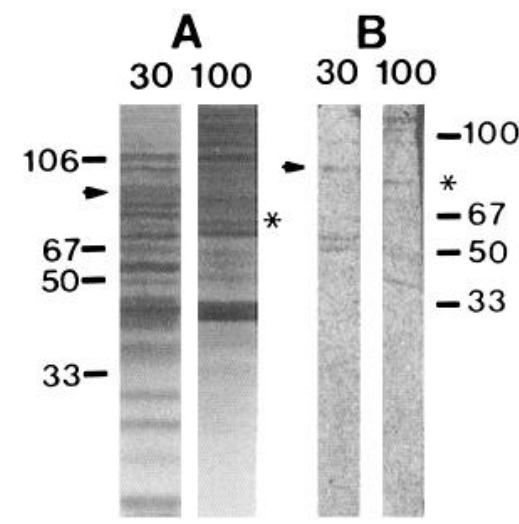

Figure 4. Identification of sp-containing proteins in fractions 3 and 4 from axoplasm. Proteins in fractions $3(30)$ and $4(100)$ were extracted into sample buffer, placed on a slab gel in duplicate, and subjected to SDS-PAGE. The gel was cut in half; one half was stained with silver $(A)$ and the other was transferred to nitrocellulose and exposed to the anti-sp antibody $(B)$. The lanes are different in length due to distortions introduced during the silver staining. Notice that the filters did not separate by size alone. The exclusion limits are based on the behavior of globular proteins, however, and proteins with other shapes can probably pass through the pores. Fraction 3 contains a stained band at 83 $\mathrm{kDa}$ (arrowhead in $A$ ) that is also recognized by the antibody (arrowhead in $B$ ). Fraction 4 has a $75 \mathrm{kDa}$ band (asterisk in $A$ ) that is also recognized by the antibody (asterisk in $B$ ). The 83 and $75 \mathrm{kDa}$ polypeptides were also recognized by the antibody in total nervous tissue (Ambron et al., 1992).

showed that fractions 3 and 4 , but not the others, contained proteins recognized by the anti-sp antibody (not shown). Thus, soluble sp-containing polypeptides are present in axoplasm. Fractions 3 and 4 contained $29 \%$ and $31 \%$, respectively, of the total extruded soluble protein.

To determine which of the sp-containing polypeptides were in these two fractions, we used SDS-PAGE, silver staining, and Western blots (Fig. 4). Soluble axoplasm contains roughly 70 polypeptides, but fractions 3 and 4 had mainly the higher-molecular-weight constituents. Fraction 3 contained an $83 \mathrm{kDa}$ polypeptide (arrow, Fig. $4 A$ ) and a prominent $75 \mathrm{kDa}$ polypeptide was present in fraction 4 (asterisk, Fig. $4 A$ ). Neither polypeptide was present in the other fraction. That these were the sp-containing constituents found previously (Ambron et al., 1992) was shown on Western blots probed using the anti-sp antibody; the $83 \mathrm{kDa}(\mathrm{sp} 83)$ and $75 \mathrm{kDa}(\mathrm{sp} 75)$ polypeptides were recognized in their respective fractions (Fig. $4 B$ ).

sp75 and sp83 are contained in the nucleus. We thought that axoplasmic sp75 and $\mathrm{sp} 83$, like exogenous HSA-sp, are capable of using the transport/import pathway and thus should be found in the nucleus. A major advantage to using large Aplysia neurons is the ability to obtain purified nuclei by manual dissection (Ambron, 1982; Elliot et al., 1993). We used the affinity-purified anti-sp antibody to probe Western blots of the soluble fraction from 60 nuclei (see Materials and Methods). Soluble axoplasm was used as a reference. As seen in Figure 5, the antibody recognized sp 75 and sp83 from both sources.

Fraction 3, which contains sp83, exhibits transport after injection. To see if any proteins in these two fractions use the transport/import pathway, the total polypeptides in each were coupled to rhodamine and injected into varicosities. None of the protein in fraction 4 moved from the injection site (not shown). In contrast, fluorescent protein in fraction 3 was transported rapidly through the axon and entered the nucleus. We

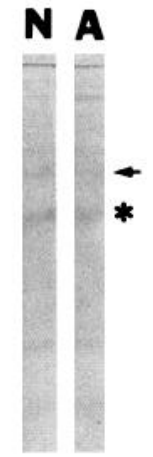

Figure 5. Sp83 and $\mathrm{sp} 75$ are present in axoplasm and the nucleus. Axoplasm extruded from 10 nerves and nuclei isolated from 60 cells by manual dissection were separated by SDS-PAGE. The polypeptides were transferred to nitrocellulose and probed with the affinity-purified anti-sp antibody. Sp83 (arrow) and sp75 (asterisk) were detected in the lanes from both the nucleus $(N)$ and axoplasm $(A)$.

injected a total of nine neurons with the fraction 3 proteins and transport/import was seen in each. A typical result is shown in Figure 6. Only a small percentage of the total rhodaminated protein reached the nucleus (Fig. $6 A, B$ ). Most remained in the varicosity, where it was both diffusely and punctately distributed (Fig. 6C,D). Interestingly, some fluorescent protein was anterogradely transported into the neurites and growth cones.

\section{The sp is required for HSA to enter the nucleus}

The data obtained using constructs and identified proteins imply that if a protein is transported, then it will also be imported into the nucleus. We wished to establish that the $\mathrm{sp}$ is required for nuclear import in these neurons. We know that rHSA (Ambron et al., 1992) and rHSA-LAp (Fig. 1C) are not transported after injection into varicosities, but instead accumulate in discrete loci near the injection site. When rHSA and rHSA-LAp were injected into the cell body, neither was imported into the nucleus (Fig. $7 A, B$ ). These results provide some additional evidence that the signals that mediate import and transport are the same.

\section{Discussion}

The retrograde transport and nuclear import processes recognize the same sps

Proteins that use the nuclear import pathway have an NLS that mediates the entry of the protein to the nucleus. The $\mathrm{sp}$ of the SV 40 large T antigen is the most widely studied NLS and is characterized by a cluster of five basic amino acids (Table 1). Many other nuclear proteins have NLSs that are homologous to the sp and comparison of eight of these signals indicates that a four amino acid sequence, -lys-arg/lys-X-arg/lys- (-K-R/K$\mathrm{X}-\mathrm{R} / \mathrm{K}-$ ), is sufficient to mediate import (Garcia-Bustos et al., 1991). Proteins enter the nucleus through the nuclear pore in a two-step process (Feldherr et al., 1984; Newmeyer and Forbes, 1988; Richardson et al., 1988) that can be blocked by WGA, a lectin that binds to O-linked glycoproteins of the pore complex (Finlay et al., 1987). The import process has been well studied in non-neuronal cells from yeast to mammals and appears to be universal (for reviews, see Goldfarb, 1989; Garcia-Bustos et al., 1991; Nigg et al., 1991; Silver, 1991). Our studies indicate that nuclear import in Aplysia neurons conforms to the general model. For example, rHSA-sp and the nuclear proteins nucleoplasmin and histone $\mathrm{H}-\mathrm{l}$ are targeted to the nucleus after mi- 

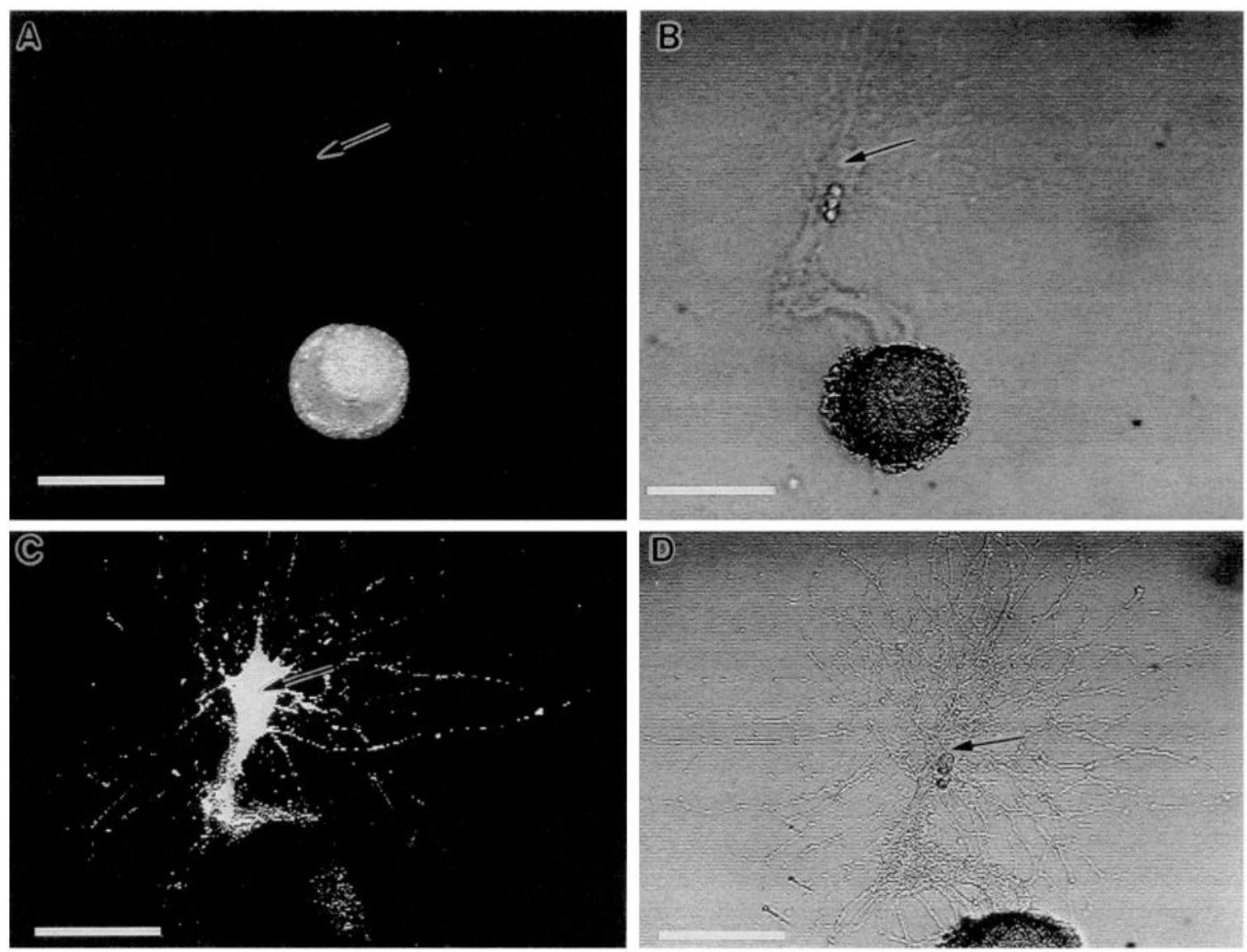

Figure 6. Soluble protein in fraction 3 from axoplasm is conveyed to the nucleus after injection. Rhodaminated proteins in fraction 3 from axoplasm were injected into varicosities and the neurons were examined $20 \mathrm{hr}$ later. The results from a typical cell are shown. $A$ and $B$, A focal plane through the middle of the soma. Fluorescent protein was transported from the injection site into the nucleus. This is a small percentage of the fluorescent protein in the cell, however. In $C$ and $D$, Taken at the level of the neurites, most of the fluorescent protein in the cell was near the injection site, where it was diffusely distributed. At low gain settings of this region, punctate fluorescence was also evident (not shown). Notice the presence of fluorescent protein in the extensive network of neurites that emerged from the varicosity. Scale bar, $250 \mu \mathrm{m}$.

croinjection into the cell body. The uptake into the nucleus is rapid and essentially complete, and occurs via a mechanism that can be blocked by prior injection of WGA (not shown).

What makes neurons unique and exciting is the presence of an axonal retrograde transport mechanism for delivery of proteins to the cell body. The combination of transport and import thereby constitutes a pathway capable of conveying proteins from the axon periphery all the way to the nucleus. An important feature of this pathway is that access to both transport and import is mediated by sp. This conclusion is based on the fact that tinkering with sp affected both processes. Thus, both processes recognized HSA-sp (Fig. $1 A$ ), but not HSA (Fig. 7 $A$; Ambron et al., 1992) or HSA-LAp (Figs. 1C, 7B; Table 1). Also, when HSA was coupled to msp, in which a lysine was replaced by threonine, the rHSA-msp construct was as ineffective in mediating retrograde transport (Ambron et al., 1992; Table 1) as nuclear import (Lanford and Butel, 1984). Finally, free sp effectively competed for access to the retrograde transporter, since when rHSA-sp and sp were co-injected the proportion of rHSA$\mathrm{sp}$ that reached the nucleus was markedly reduced (Fig. $1 B$ ).

The ability of sp to compete with HSA-sp points to a saturable receptor in Aplysia nerves. We have examined the soluble fraction of extruded axoplasm and identified two polypeptides of approximately 60 and $67 \mathrm{kDa}$ that bind specifically to a column of immobilized sp (M. Smith, R. Schmied, R. T. Ambron, un- published observations). These putative $\mathrm{sp}$ receptors are likely homologs of the approximately 59 and $69 \mathrm{kDa} s p$ receptors found in the cytoplasm of other cell types (Garcia-Bustos et al., 1991) and would constitute another feature shared by both the import and transport processes. The cytoplasmic receptors are thought to facilitate the translocation of sp-containing proteins from the cytoplasm into the nucleus, but the details are not known (Nigg et al., 1991). Homology between the cytoplasmic and axonal receptors would also indicate that the movement of NLS proteins from the cytoplasm to the nucleus is an active, vectorial process that is homologous to retrograde transport through the axon. Consistent with this idea is our observation that proteins that reach the cell body from the axon seem to go directly to the nucleus without disseminating throughout the cytoplasm. Thus, defining the relationship between the $\mathrm{sp}$, its receptor, and the retrograde transport system might provide insight into the movement of nuclear proteins in non-neuronal cells.

One way to characterize the receptor proteins is to assume that transport efficiency reflects the affinity of receptor binding. If we correlate our previous studies using msp, mentioned above, with our recent findings, we can rank the proteins according to their ability to be transported: HSA-sp $\geq$ nucleoplasmin $\gg$ histone $\mathrm{H}-1$ > HSA-msp > HSA (Table 1). We would expect these constituents to bind to the isolated receptor in the same 

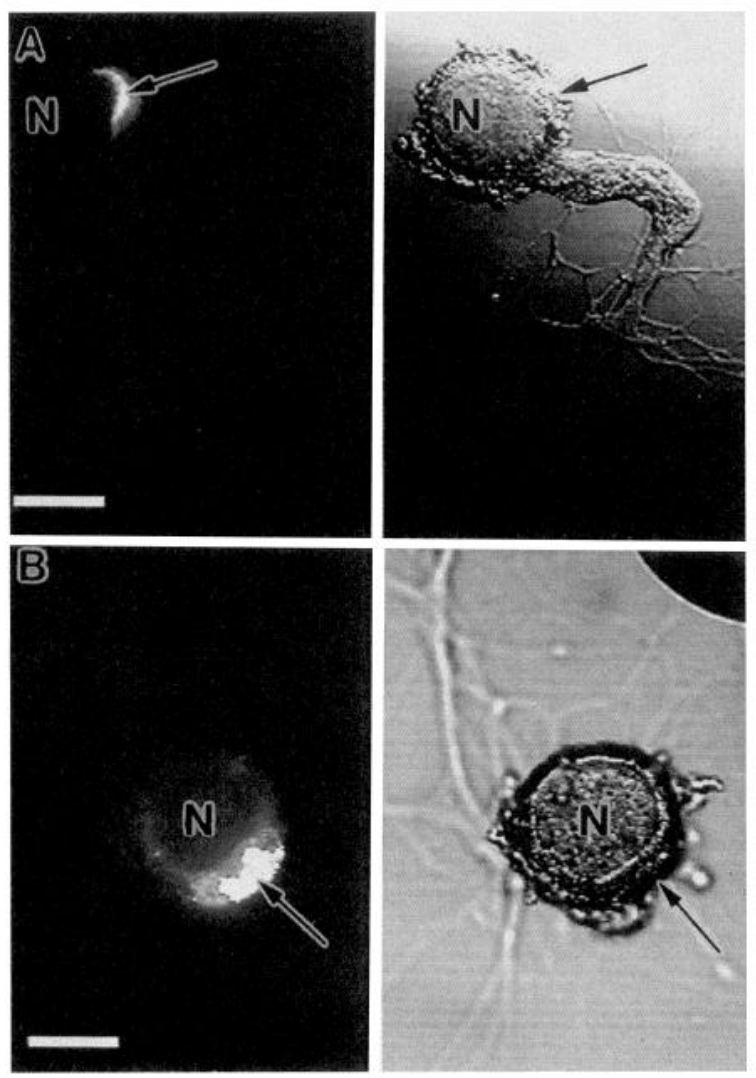

Figure 7. rHSA and rHSA-LAp do not enter the nucleus $(N)$ after microinjection into the cell body. Neurons were injected (arrow) with (A) rHSA, or $(B)$ rHSA-LAp, and $3 \mathrm{hr}$ later the cells were fixed and examined by confocal microscopy. Both constructs remained in the cytoplasm. Scale bar, $100 \mu \mathrm{m}$.

order. This hierarchy also provides some insight into the requirements for binding to the receptor. Nucleoplasmin has two NLSs located in the C-terminal tail region (Table 1; Dingwall et al., 1988). One, -ala-lys-lys-lys-lys-, is similar to the minimal NLS of the sp described above. The NLS of calf thymus histone $\mathrm{H}-1$ has not been conclusively identified, but the protein was actively imported when microinjected into kidney epithelial cells (Breeuwer and Goldfarb, 1990) and it contains a -pro-lyslys-ala-lys-lys- sequence that is conserved in rabbit and trout (Van Holde, 1989). Also, the presence of a proline adjacent to a group of basic amino acids is a motif found in several NLSs (Garcia-Bustos et al., 1991). If we assume that these sequences are recognized by the retrograde transport apparatus, then transport, like nuclear import, requires a cluster of at least four basic amino acids and substitution of lysine for arginine is acceptable. These findings provide direction for future research and must be interpreted cautiously since it has proven extraordinarily difficult to analyze the contributions made by the various components of an NLS (Lanford and Butel, 1986; Lobl et al., 1990; Garcia-Bustos et al., 1991).

\section{Endogenous axoplasmic proteins use the transport/import pathway}

The presence of receptors for $\mathrm{sp}$ in axoplasm and the fact that exogenous sp-conjugated proteins and constructs are transported prompted a search for endogenous proteins that use the transport/import pathway. We used four criteria to identify such proteins. The proteins would (1) be present in the soluble frac- tion of axoplasm, (2) be present in the nucleus, (3) contain sp, and (4) be transported/imported when injected into the axon. We wish to point out that these criteria are restrictive and would cause us to miss some proteins. For example, proteins that enter the nucleus and remain there for only a short time might not be detected and therefore would not meet the second criterion. Also, isolated proteins in which the $\mathrm{sp}$ is not exposed (see below) will not be transported or imported. Nevertheless, we felt that by being stringent we would identify those proteins that were most likely to use the pathway.

So far, $\mathrm{sp} 83$ meets at least the first three criteria. $\mathrm{Sp} 83$ was previously identified on Western blots of soluble Aplysia nervous tissue (Ambron et al., 1992). In a refinement of these earlier studies, we now found sp 83 in fraction 3 from soluble extruded axoplasm (Fig. 4) and also in the nucleus (Fig. 5). When fraction 3 was injected into the varicosities of nine cells, in each case one or more proteins used the transport/import pathway to the nucleus (Fig. 6). Fraction 3 contains 25 polypeptides, but we reasoned that the retrograde transport machinery would recognize the sp-containing constituents and convey them to the cell body. Since $\operatorname{sp} 83$ is found in the nucleus and is the major, if not the only, sp-containing polypeptide in fraction 3 , it is reasonable to assume that this constituent was transported/imported. If correct, then $\mathrm{sp} 83$ must have been transport competent at the time of its isolation (see below). Direct evidence to support these ideas awaits the isolation of this protein.

A $75 \mathrm{kDa}$ polypeptide (sp75) meets three of the criteria: it contains sp and is found in the axoplasm and nucleus (Figs. 4, 5). It was interesting, therefore, that fraction 4 from the axoplasm, which contains this protein, did not show transport after injection. As discussed below, this would be explained if the $\mathrm{sp}$ was masked through an association with another protein. The fact that $\mathrm{sp} 75$ was present in the $>100 \mathrm{kDa}$ fraction supports this idea. Alternatively, the mere presence of the sp sequence may not be sufficient to cause transport. What argues against this is the fact that sp75 is in both the nucleus and axoplasm. Clearly, we need to know more about this constituent.

Other proteins in these fractions behaved as expected of resident axoplasmic proteins that were removed and then inserted back into the axon; some moved in the anterograde direction into the neurites and growth cones, some remained in the axoplasm, while others were taken up into organelles (Fig. $6 C, D$ ), probably to be destroyed (R. Schmied and R. T. Ambron, unpublished observations). The anterogradely transported material is interesting since it presumably moved by associating with the surface of vesicles. Synapsin I is transported this way and a synapsin I homolog is present in Aplysia neurons (Bongiovi et al., 1992).

We are actively attempting to isolate $\operatorname{sp} 83$ for injection. It is relevant, therefore, that an $83 \mathrm{kDa}$ soluble glycoprotein, with single O-linked $\mathrm{N}$-acetylglucosamine moieties, has been found in both the axoplasm and nucleus of Aplysia neurons (Elliot et al., 1993). Preliminary comparisons between the two suggest that this glycoprotein and $\operatorname{sp} 83$ are one and the same. Since $\mathrm{O}$-linked $\mathrm{N}$-acetylglucosamine is a conserved characteristic of several transcription factors in the nucleus and is present on a variety of proteins involved in regulating protein synthesis (see Elliot et al., 1993, for references), identity would strengthen the argument that axoplasmic sp 83 is destined for transport to the nucleus.

One of our goals is to characterize endogenous proteins that use the transport/import pathway, but there are alternatives to isolating $\mathrm{sp} 83$ and $\mathrm{sp} 75$. Another approach is to identify proteins 
that are relatively abundant and have the properties expected of constituents that use the pathway. According to our hypothesis (Ambron et al., 1992), the signal sequence in such proteins is masked to allow the protein to enter the axon. Masking could occur, for example, by forming a complex with another protein (Nigg et al., 1991). According to this scenario, the $\mathrm{sp}$ would become exposed by having the complex dissociate in response to a transduction event (Ambron et al., 1992). Such events include nerve injury (Cragg, 1970; Walters et al., 1991), the binding of a trophic factor that influences neuronal phenotype or survival (Ambron et al., 1985; Macagno et al., 1986; Schotzinger and Landis, 1990; Oppenheim, 1991; French and Kristan, 1992), or the binding of a ligand that produces long-term structural changes at the presynaptic terminal (Nguyen and Atwood, 1992) such as occurs in learning and memory (Bailey and Chen, 1983).

One protein that has these properties is the catalytic subunit of the cAMP-dependent protein kinase II; this subunit is abundant, is readily isolated from mammalian brain (Welch and Fcramisco, 1985), and has a conserved sp-like sequence (Beushausen et al., 1988) that is functional, since studies in nonneuronal cells show that it enters the nucleus when the levels of cAMP are elevated (Nigg et al., 1985; Meinkoth et al., 1990). Moreover, kinase II is present in the presynaptic terminal at certain Aplysia synapses. Since the catalytic subunit separates from the regulatory subunit during learning, it would provide a convenient link between the nucleus and events that increase cAMP at the terminal (Greenberg et al., 1987).

\section{References}

Ambron RT (1982) Differences in the distribution of specific glycoproteins among the regions of a single identified neuron. Brain Res 239:489-505.

Ambron RT, Den H, Schacher S (1985) Synaptogenesis by single identified neurons in vitro: contribution of rapidly transported and newly synthesized proteins. J Neurosci 5:2857-2865.

Ambron RT, Schmied R, Huang CC, Smedman M (1992) A signal peptide mediates the retrograde transport of proteins through the axon to the cell body and into the nucleus. J Neurosci 12:2813-2818.

Bailey CH, Chen M (1983) Morphological basis of long-term habituation and sensitization in Aplysia. Science 220:91-93.

Beushausen S, Bergold P, Sturner S, Elste A, Roytenberg V, Schwartz JH, Bayley H (1988) Two catalytic subunits of cAMP-dependent protein kinase generated by alternative RNA splicing are expressed in Aplysia neurons. Neuron 1:853-864.

Bongiovi ME, Ambron RT, Silverman A-J (1992) Morphological and biochemical evidence for a synapsin I like homologue in the nervous system of Aplysia californica. J Neurosci Res 32:395-406.

Breeuwer M, Goldfarb DS (1990) Facilitated nuclear transport of histone $\mathrm{Hl}$ and other small nucleophilic proteins. Cell 60:999-1008.

Cragg BG (1970) What is the signal for chromatolysis? Brain Res 23: $1-21$.

Dingwall C, Robbins J, Dilworth SM, Roberts B, Richardson WD (1988) The nucleoplasmin nuclear location sequence is larger and more complex than that of SV-40 large T-antigen. J Cell Biol 107:841-849.

Dworetzky SI, Lanford RE, Feldherr CM (1988) The effects of variations on the number and sequence of targeting signals on nuclear uptake. J Cell Biol 107:1279-1287

Elliot SP, Schmied R, Gabel CA, Ambron RT (1993) An 83 kDa O-GIcNAc-glycoprotein is found in the axoplasm and nucleus of $A p l y$ sia neurons. J Neurosci 13:2424-2429.

Feldherr CM, Kallenbach E, Schultz N (1984) Movement of a karyophilic protein through the nuclear pores of oocytes. J Cell Biol 99: 2216-2222.

Finlay DR, Newmeyer DD, Price TM, Forbes DJ (1987) Inhibition of in vitro nuclear transport by a lectin that binds to nuclear pores. $J$ Cell Biol 104:189-200.

French KA, Kristan WB Jr (1992) Target influence on the development of leech neurons. Trends Neurosci 15:169-174.
Garcia-Bustos J, Heitman J, Hall MN (1991) Nuclear protein localization. Biochcm Biophys Acta 1071:83-101.

Goldfarb DS (1989) Nuclear transport. Curr Opin Cell Biol 1:441446.

Goldfarb DS, Gariepy L, Schoolnik JG, Kornberg RD (1986) Synthetic peptides as nuclear localization signals. Nature 322:641-644.

Graham RC, Karnovsky MJ (1966) The early stage of absorption of injected horseradish peroxidase in the proximal tubules of the mouse kidney. J Histochem Cytochem 14:291-309.

Greenberg SM, Castellucci VF, Bayley H, Schwartz JH (1987) A molecular mechanism for long-term sensitization in Aplysia. Nature 329: 62-65.

Laemmli UK (1970) Cleavage of structural proteins during the assembly of the head of bacteriophage T4. Nature 277:680-685.

Lanford RE, Butel JS (1984) Construction and characterization of an SV 40 mutant defective in nuclear transport of $T$ antigen. Cell 37: 801-813.

Lanford RE, Kanda P, Kennedy RC (1986) Induction of nuclear transport with a synthetic peptide homologous to the SV40 $\mathrm{T}$ antigen transport signal. Cell 46:575-582.

Lanford RE, Feldherr CM, White RG, Dunham RG, Kanda P (1990) Comparison of diverse transport signals in synthetic peptide-induced nuclear transport. Exp Cell Res 186:32-38.

Lobl TJ, Mitchell MA, Maggiora LL (1990) SV40 large T antigen nuclear signal analogues: successful nuclear targeting with bovine serum albumin but not low molecular weight fluorescent conjugates. Biopolymers 29:197-203.

Macagno E, Peinado A, Stewart R (1986) Segmental differentiation of the leech nervous system: specific phenotypic changes associated with ectopic targets. Proc Natl Acad Sci USA 83:2746-2750.

Meinkoth JL, Ji Y, Taylor S, Feramisco JR (1990) Dynamics of the distribution of cAMP-dependent protein kinase in living cells. Proc Natl Acad Sci USA 87:9595-9599.

Newmeyer DD, Forbes DJ (1988) Nuclear import can be separated into distinct steps in vitro: nuclear pore binding and translocation. Cell 52:641-653.

Newmeyer DD, Finlay DR, Forbes DJ (1986) In vitro transport of a fluorescent nuclear protein and exclusion of non-nuclear proteins. $J$ Cell Biol 103:2091-2102.

Nguyen PV, Atwood HL (1992) Maintenance of long-term adaptation of synaptic transmission requires axonal transport following induction in an identified crayfish motor neuron. Exp Neurol 125:414-422.

Nigg EA, Hilz H, Eppenberge HM, Dutly F (1985) Rapid and reversible translocation of the catalytic subunit of cAMP-dependent protein kinase type II from the Golgi complex to the nucleus. EMBO J 4:2801-2806.

Nigg EA, Baeuerle PA, Luhrmann R (1991) Nuclear import-export: in search of signals and mechanisms. Cell 66:15-22.

Oppenheim RW (1991) Cell death during development of the nervous system. Annu Rev Neurosci 14:453-501.

Richardson WD, Mills AD, Dilworth SM, Laskey RA, Dingwall C (1988) Nuclear protein migration involves two steps: rapid binding at the nuclear envelope followed by slower translocation through nuclear pores. Cell 52:655-664.

Schacher S, Proshanski E (1983) Neurite regeneration by Aplysia ncurons in dissociated cell culture: modulation by Aplysia hemolymph and the presence of the initial axonal segment. J Neurosci 3:24032413.

Schotzinger RJ, Landis SC (1990) Acquisition of cholinergic and peptidergic properties by sympathetic innervation of rat sweat glands requires interaction with normal targets. Neuron 5:91-100.

Sherbany AA, Ambron RT, Schwartz JH (1979) Membrane glycolipids: regional synthesis and axonal transport in a single identified neuron of Aplysia californica. Science 203:78-81.

Silver P (1991) How proteins enter the nucleus. Cell 64:489-497.

Vallee RB, Bloom GS (1991) Mechanisms of fast and slow axonal transport. Annu Rev Neurosci 14:59-92.

Van Holde KE (1989) Chromatin. New York: Springer.

Walters T, Alizadeh H, Castro CA (1991) Similar neuronal altcration induced by axonal injury and learning in Aplysia. Science 253:797799.

Welch WJ, Feramisco JR (1985) Rapid purification of mammalian 70,000-dalton stress proteins: affinity of the proteins for nucleotides. Mol Cell Biol 5:1229-1237. 